\title{
A NOTE ON GENERIC CLIFFORD ALGEBRAS OF BINARY CUBIC FORMS
}

\author{
LINHONG WANG AND XINGTING WANG*
}

\begin{abstract}
We study the representation theoretic results of the binary cubic generic Clifford algebra $\mathcal{C}$, which is an Artin-Schelter regular algebra of global dimension five. In particular, we show that $\mathcal{C}$ is a PI algebra of PI degree three and compute its point variety and discriminant ideals. As a consequence, we give a necessary and sufficient condition on a binary cubic form $f$ for the associated Clifford algebra $\mathcal{C}_{f}$ to be an Azumaya algebra.
\end{abstract}

\section{INTRODUCTION}

Let $f$ be a form of degree $m$ in $n$ variables over a base field $k$. The Clifford algebra $\mathcal{C}_{f}$ associated to the form $f$ is defined to be an associative algebra $k\left\langle x_{1}, \ldots, x_{n}\right\rangle$ subject to the relations $\left(a_{1} x_{1}+\cdots+a_{n} x_{n}\right)^{m}-f\left(a_{1}, \ldots, a_{n}\right)$ for all $a_{1}, \ldots, a_{n} \in k$. In [10], Chan-Young-Zhang defined the generic Clifford algebra (or more precisely universal Clifford algebra) $\mathcal{C}_{m, n}=k\left\langle x_{1}, \ldots, x_{n}\right\rangle$ subject to the relations $\left(a_{1} x_{1}+\cdots+\right.$ $\left.a_{n} x_{n}\right)^{m} x_{j}-x_{j}\left(a_{1} x_{1}+\cdots+a_{n} x_{n}\right)^{m}$ for all $a_{1}, \ldots, a_{n} \in k$ and $1 \leq j \leq n$. The work of $\$ 2.6$ implies that the Clifford algebra $\mathcal{C}_{f}$ is a homomorphic image of the generic Clifford algebra $\mathcal{C}_{m, n}$, which paves a way to understand the representations of the former through the representations of the latter. Chan-Young-Zhang further showed that ([10, Lemma 3.8]) when $m=2, n=2$, the binary quadratic generic Clifford algebra belongs to the nice family of connected graded algebras that are Artin-Schelter regular (see [2]).

In this note, we focus on the binary cubic generic Clifford algebra $(m=3, n=2)$ and denote it by $\mathcal{C}$. In [19], all Artin-Schelter regular algebras of global dimension five that are generated by two generators with three generating relations are classified. The algebra $\mathcal{C}$ is listed as one of the type $\mathbf{A}$ algebras there, which is also proved to be strongly noetherian, Auslander regular and Cohen-Macaulay. In this note, we further show that $\mathcal{C}$ is indeed a polynomial identity (PI) algebra of PI degree three (see Theorem 4.4). This and other known properties allow us to fully understand the representations of $\mathcal{C}$ by the well-developed representation theory of PI algebras (e.g. [7, 6, 8]). As a consequence, we derive some ring-theoretic results of the binary cubic

2010 Mathematics Subject Classification. 16G30, 16R99.

Key words and phrases. Clifford algebra, point variety, discriminant ideals.

*corresponding author. 
Clifford algebra $\mathcal{C}_{f}$, whose properties have been studied by Heerema [14 and Haile [12].

Acknowledgement. This work was inspired by the poster presentation given by Charlotte Ure at Algebra Extravaganza! at Temple University in July 2017. The authors also learned from Q.-S. Wu later that the binary cubic generic Clifford algebra has appeared as one of the type $\mathbf{A}$ algebras in the classification of global dimensional five Artin-Schelter regular algebras generated by two generators with three generating relations; see [19]. The authors are grateful for the correspondence from them. The authors also want to thank the referee for his/her careful reading and suggestions.

\section{BACKGROUND}

2.1. Let $k$ be an algebraically closed base field of characteristic not 2 or 3 . The reader is referred (e.g.) to [11] and [15] for further background on the theory of PI rings. Recall that a ring $R$ with center $Z(R)$ is called Azumaya over $Z(R)$ if $R$ is a finitely generated projective $Z(R)$-module and the natural map $R \otimes_{Z(R)} R^{\mathrm{op}} \rightarrow \operatorname{End}_{\mathrm{Z}(\mathrm{R})}(\mathrm{R})$ is an isomorphism. Let $\Lambda$ be a prime noetherian affine $k$-algebra finitely generated as a module over its center $Z$. Define

$$
\begin{aligned}
\mathcal{A}_{\Lambda}: & =\left\{\mathfrak{m} \in \operatorname{maxSpec}(Z) \mid \Lambda_{\mathfrak{m}} \text { is Azumaya over } Z_{\mathfrak{m}}\right\}, \\
\mathcal{S}_{\Lambda}: & =\left\{\mathfrak{m} \in \max \operatorname{Spec}(Z) \mid Z_{\mathfrak{m}} \text { is not regular }\right\} .
\end{aligned}
$$

The set $\mathcal{A}_{\Lambda}$ is called the Azumaya locus of $\Lambda$ over $Z$, and $\mathcal{S}_{\Lambda}$ is the singular locus of $\Lambda$ (or of $Z$ ).

2.2. Theorem. [6, Theorem III.1.7][7, Proposition 3.1, Lemma 3.3] Let $\Lambda$ be a prime noetherian affine $k$-algebra that is module-finite over its center $Z$.

(a) The maximum $k$-dimension of irreducible $\Lambda$-modules equals the PI degree of $\Lambda$.

(b) Let $S$ be an irreducible $\Lambda$-module, $P=\operatorname{Ann}_{\Lambda}(\mathrm{S})$, and $\mathfrak{m}=P \cap Z$. Thus $\operatorname{dim}_{k}(S)=\mathrm{PI}-\operatorname{deg}(\Lambda)$ if and only if $\Lambda_{\mathfrak{m}}$ is Azumaya over $Z_{\mathfrak{m}}$.

Moreover if gldim $(\Lambda)<\infty$, then $\mathcal{A}_{\Lambda}$ is a nonempty open (and hence dense) subset of $\operatorname{maxSpec}(\mathrm{Z}) \backslash \mathcal{S}_{\Lambda}$.

2.3. In [10], Chan-Young-Zhang defined the generic Clifford algebra and established some properties in the quadratic case. Recall that the binary cubic generic Clifford algebra $\mathcal{C}$ is defined as the quotient algebra of the free algebra $k\langle x, y\rangle$ subject to the relations:

$$
x^{3} y-y x^{3}, x^{2} y^{2}+x y x y-y x y x-y^{2} x^{2}, x y^{3}-y^{3} x .
$$

Moreover, $\mathcal{C}$ is one of the type $\mathbf{A}$ algebras studied in [19] (parameters $t=-1, l_{2}=1$ ).

2.4. Theorem. [19, Theorem 5] The connected graded algebra $\mathcal{C}$ is Artin-Schelter regular of global dimension five. Moreover, $\mathcal{C}$ is an Auslander regular, Cohen-Macaulay, and strongly noetherian domain. 
2.5. By the diamond lemma [5], we have that

$$
\left\{y^{i}\left(x y^{2}\right)^{j}(x y)^{k}\left(x^{2} y\right)^{l} x^{m} \mid i, j, k, l, m \in \mathbb{N}\right\}
$$

is a $k$-linear basis for $\mathcal{C}$. Consequently, the Hilbert series of $\mathcal{C}$ is $\frac{1}{(1-t)^{5}(1+t)\left(1+t+t^{2}\right)^{2}}$.

2.6. Let $f(u, v)=a u^{3}+3 b u^{2} v+3 c u v^{2}+d v^{3}$ be a cubic form with $a, b, c, d \in k$. Its discriminant is given by

$$
D:=\frac{1}{4}(a d-b c)^{2}-\left(a c-b^{2}\right)\left(b d-c^{2}\right) .
$$

The Clifford algebra $\mathcal{C}_{f}$ of the cubic form $f$ is defined as

$$
\mathcal{C}_{f}:=k\langle x, y\rangle / I,
$$

where $I$ is the ideal generated by the elements $(u x+v y)^{3}-f(u, v)$ for all $u, v \in k$. It is easy to see that

$$
I=\left(x^{3}-a, y^{3}-d, x^{2} y+x y x+y x^{2}-3 b, y^{2} x+y x y+x y^{2}-3 c\right) .
$$

There is a natural surjection $\mathcal{C} \rightarrow \mathcal{C}_{f}$ by sending $x \mapsto x, y \mapsto y$ since the ideal generated by the defining relations in Eq.(1) for $\mathcal{C}$ is contained in $I$.

2.7. Theorem. [12, Theorem 1.1', Corollary 1.2'] The Clifford algebra $\mathcal{C}_{f}$ is an Azumaya algebra of PI degree three if $D \neq 0$. In this case, its center is a Dedekind domain, isomorphic to the coordinate ring of the affine elliptic curve $u^{2}=v^{3}-27 D$.

\section{The Center and Singular locus of $\mathcal{C}$}

3.1. Consider the projective space $\mathbb{P}^{3}$ with homogenous coordinates $\left[z_{0}: z_{1}: z_{2}: z_{3}\right]$. For cubic binary forms, the discriminant projective variety is defined by $\Delta=0$, where (see Eq.(2) )

$$
\Delta=\frac{1}{4}\left(z_{0} z_{3}-z_{1} z_{2}\right)^{2}-\left(z_{0} z_{2}-z_{1}^{2}\right)\left(z_{1} z_{3}-z_{2}^{2}\right) .
$$

3.2. Lemma. The reduced variety of the singular locus of the discriminant projective variety is given by the twisted cubic curve $v: \mathbb{P}^{1} \rightarrow \mathbb{P}^{3}$ via

$$
v:\left[x_{0}: x_{1}\right] \mapsto\left[x_{0}^{3}: x_{0}^{2} x_{1}: x_{0} x_{1}^{2}: x_{1}^{3}\right]=\left[z_{0}: z_{1}: z_{2}: z_{3}\right] .
$$


Proof. It is well known (e.g. [13, Chapter I Theorem 5.1]) that the singular locus of $\Delta=0$ is the zero locus of the polynomials

$$
\begin{aligned}
& \frac{\partial \Delta}{\partial z_{0}}=-\frac{3}{2} z_{1} z_{2} z_{3}+\frac{1}{2} z_{0} z_{3}^{2}+z_{2}^{3}=0, \\
& \frac{\partial \Delta}{\partial z_{1}}=-\frac{3}{2} z_{0} z_{2} z_{3}-\frac{3}{2} z_{1} z_{2}^{2}+3 z_{1}^{2} z_{3}=0, \\
& \frac{\partial \Delta}{\partial z_{2}}=-\frac{3}{2} z_{0} z_{1} z_{3}-\frac{3}{2} z_{1}^{2} z_{2}+3 z_{0} z_{2}^{2}=0, \\
& \frac{\partial \Delta}{\partial z_{3}}=-\frac{3}{2} z_{0} z_{1} z_{2}+\frac{1}{2} z_{0}^{2} z_{3}+z_{1}^{3}=0 .
\end{aligned}
$$

If $z_{0}=0$, then $z_{1}=z_{2}=0$ and $z_{3} \neq 0$, which corresponds to the point $v([0: 1])$. Now let $z_{0}=1$. From Eq.(77), we have $z_{3}=3 z_{1} z_{2}-2 z_{1}^{3}$. Substituting it into Eq.(6), we obtain $\left(z_{1}^{2}-z_{2}\right)^{2}=0$ which implies that $z_{2}=z_{1}^{2}$ and $z_{3}=z_{1}^{3}$. One can check that $\left[1: z_{1}: z_{1}^{2}: z_{1}^{3}\right]=v\left(\left[1: z_{1}\right]\right)$ satisfies Eq.(44)-Eq.(7). Hence the solutions are exactly given by the twisted cubic curve.

3.3. In the following, we denote by $Z$ the center of the generic Clifford algebra $\mathcal{C}$. We will describe the center $Z$ according to [18]. Consider the following central elements of $\mathcal{C}$, where $\omega \in k$ is a primitive third root of unity.

$$
\left\{\begin{aligned}
z_{0} & =x^{3} \\
z_{1} & =\frac{1}{3}\left(x^{2} y+x y x+y x^{2}\right) \\
z_{2} & =\frac{1}{3}\left(y^{2} x+y x y+x y^{2}\right) \\
z_{3} & =y^{3} \\
z_{4} & =(y x-\omega x y)^{3}-\frac{3}{2} \omega(1-\omega) x^{3} y^{3}-\frac{9}{2}\left(1+2 \omega^{2}\right) z_{1} z_{2} \\
z_{5} & =(x y)^{2}-y^{2} x^{2}=(y x)^{2}-x^{2} y^{2}
\end{aligned}\right.
$$

Define the formal discriminant element $\Delta$ in $\mathcal{C}$ as

$$
\Delta=\frac{1}{4}\left(z_{0} z_{3}-z_{1} z_{2}\right)^{2}-\left(z_{0} z_{2}-z_{1}^{2}\right)\left(z_{1} z_{3}-z_{2}^{2}\right) .
$$

Under the natural surjection $\mathcal{C} \rightarrow \mathcal{C}_{f}$, it follows that $\left(z_{0}, z_{1}, z_{2}, z_{3}\right) \mapsto(a, b, c, d)$. In particular, the formal discriminant element $\Delta$ maps to the discriminant $D \in k$ of the binary cubic form $f$ under the surjection $\mathcal{C} \rightarrow \mathcal{C}_{f}$.

3.4. Theorem. [18] The generic Clifford algebra $\mathcal{C}$ is finitely generated as a module over its center $Z$. Moreover, the center $Z$ is generated by $\left(z_{i}\right)_{0 \leq i \leq 5}$ subject to one relation $z_{4}^{2}=z_{5}^{3}-27 \Delta$. Consequently, $\operatorname{maxSpec}(Z)$ is isomorphic to the coordinate ring of a relative quasiprojective curve over the 4-dimensional affine space $\mathbb{A}^{4}=$ $\operatorname{maxSpec}\left(k\left[z_{0}, z_{1}, z_{2}, z_{3}\right]\right)$ that is elliptic over an open subset of $\mathbb{A}^{4}$. 
3.5. Corollary. The reduced variety of the singular locus $\mathcal{S}_{\mathcal{C}}$ of $Z$ is an affine twisted cubic curve in $\mathbb{A}^{4}=\operatorname{maxSpec}\left(k\left[z_{0}, z_{1}, z_{2}, z_{3}\right]\right)$ defined by

$$
\mathcal{S}_{C}=\mathbb{V}\left(z_{4}, z_{5}, z_{0} z_{3}-z_{1} z_{2}, z_{0} z_{2}-z_{1}^{2}, z_{1} z_{3}-z_{2}^{2}\right) .
$$

Proof. Since the center $Z$ generated by $\left(z_{i}\right)_{0 \leq i \leq 5}$ is subject to one relation $R:=z_{4}^{2}-$ $z_{5}^{3}+27 \Delta$, the singular locus $\mathcal{S}_{\mathcal{C}}$ of $Z$ is the zero locus of the derivatives $\frac{\partial R}{\partial z_{i}}$ for $0 \leq i \leq 5$, or equivalently (if ignoring multiplicity) $z_{4}=z_{5}=0$ and $\frac{\partial \Delta}{\partial z_{0}}=\frac{\partial \Delta}{\partial z_{1}}=\frac{\partial \Delta}{\partial z_{2}}=\frac{\partial \Delta}{\partial z_{3}}=0$. Thus, the result follows from Lemma 3.2 .

\section{Irreducible Representations and Azumaya locus of $\mathcal{C}$}

4.1. It is clear that the set of isomorphism classes of one-dimensional representations of $\mathcal{C}$ is bijective to $\mathbb{A}^{2}$ via the one-to-one correspondence $\operatorname{Ann}_{\mathcal{C}}(S)=(x-a, y-b) \leftrightarrow$ $(a, b) \in \mathbb{A}^{2}$ for any $\mathcal{C}$-module $S$ with $\operatorname{dim}_{k}(S)=1$. The following result is crucial to our work.

\subsection{Lemma. There are no two-dimensional irreducible representations over $\mathcal{C}$.}

Proof. Suppose $S$ is any two-dimensional irreducible representation over $\mathcal{C}$. This implies that there is a surjective algebra map $\varphi: \mathcal{C} \rightarrow \operatorname{End}_{k}(S)=\mathrm{M}_{2}(k)$. One sees easily that $\varphi(x) \neq 0$; otherwise $S$ can be viewed as a module over $\mathcal{C} /(x) \cong k[y]$, where all irreducible representations are one-dimensional. The same argument shows that $\varphi(x)$ is not a scalar multiple of the identity matrix in $\mathrm{M}_{2}(k)$. Since $x^{3}$ is central in $\mathcal{C}, \varphi\left(x^{3}\right)=\varphi(x)^{3}$ is a scalar multiple of the identity matrix. Therefore by a linear transformation of $S$ and a possible rescaling of the variables $x, y$ of $\mathcal{C}$, we can assume that $\varphi(x)=\left(\begin{array}{ll}1 & 0 \\ 0 & \omega\end{array}\right)$ where $\omega$ is a primitive third root of unity. Now write $\varphi(y)=\left(\begin{array}{ll}a & b \\ c & d\end{array}\right)$ for some $a, b, c, d \in k$. When we apply $\varphi$ to the relations in $\mathcal{C}$, we obtain

$$
\varphi\left(x^{2} y^{2}+(x y)^{2}-(y x)^{2}-y^{2} x^{2}\right)=\left(\begin{array}{cc}
0 & 3 b\left(a-\omega^{2} d\right) \\
-3 c\left(a-\omega^{2} d\right) & 0
\end{array}\right)=0 .
$$

If $a \neq \omega^{2} d$, then we have $b=c=0$. So $\varphi(x)$ and $\varphi(y)$ are both diagonal. This shows that $S$ is not irreducible, which is a contradiction. Hence we have $a=\omega^{2} d$. Thus, $\varphi$ sends the central element $y^{3}$ to

$$
\varphi\left(y^{3}\right)=\left(\begin{array}{cc}
a^{3}+(2+\omega) a b c & b^{2} c \\
b c^{2} & a^{3}+(1+2 \omega) a b c
\end{array}\right) .
$$

Since $\varphi\left(y^{3}\right)$ is a scalar multiple of the identity matrix, we obtain $b c=0$ and $\varphi(y)$ is either upper or lower triangular. Again, it is a contradiction since $S$ is irreducible.

4.3. We denote by $\operatorname{Irr} \mathcal{C}$ the isomorphism classes of all irreducible representations over $\mathcal{C}$. For each possible integer $n \geq 1, \operatorname{Irr}_{n} \mathcal{C}$ denotes the isomorphism subclasses of all 
$n$-dimensional irreducible representations over $\mathcal{C}$. Recall $\mathcal{S}_{\mathcal{C}} \subset \max \operatorname{Spec}(Z)$ is the singular locus of $\mathcal{C}$ and we call its complement $\operatorname{maxSpec}(Z) \backslash \mathcal{S}_{\mathcal{C}}$ the smooth locus of $\mathcal{C}$. Note that there is a natural surjection

$$
\chi: \operatorname{Irr} \mathcal{C} \rightarrow Y:=\max \operatorname{Spec}(Z)
$$

via $S \mapsto \operatorname{Ann}_{\mathcal{C}}(S) \cap Z$ for any irreducible representation $S$ over $\mathcal{C}$.

4.4. Theorem. The following hold for the binary cubic generic Clifford algebra $\mathcal{C}$.

(a) $\mathcal{C}$ is a PI algebra of PI degree three.

(b) $\operatorname{Irr} \mathcal{C}=\operatorname{Irr}_{1} \mathcal{C} \sqcup \operatorname{Irr}_{3} \mathcal{C}$.

(c) The map $\operatorname{Irr}_{1} \mathcal{C} \rightarrow S_{\mathcal{C}}$ is three to one.

(d) The map $\operatorname{Irr}_{3} \mathcal{C} \rightarrow \operatorname{maxSpec}(Z) \backslash \mathcal{S}_{\mathcal{C}}$ is one to one.

(e) The Azumaya locus of $\mathcal{C}$ coincides with the smooth locus of $\mathcal{C}$.

Proof. (a) By Theorem 3.4, we know $\mathcal{C}$ is module-finite over its center $Z$ and hence is PI by [15, Corollary 13.1.13(iii)]. Now suppose $\mathcal{C}$ has PI degree $n$. Regarding Eq.(10), we know $\chi\left(\operatorname{Irr}_{n} \mathcal{C}\right)$ is an open dense subset of $Y$ by Theorem 2.2. Take the formal discriminant $\Delta$ of $\mathcal{C}$ as in Eq.(9). Note that $Y \backslash \mathbb{V}(\Delta)$ is another open dense subset of $Y$ since $Y$ is irreducible. So there exists a maximal ideal

$$
\mathfrak{m}=\left(z_{0}-a, z_{1}-b, z_{2}-c, z_{3}-d, z_{4}-e, z_{5}-f\right) \in \chi\left(\operatorname{Irr}_{n} \mathcal{C}\right) \backslash \mathbb{V}(\Delta),
$$

for some $a, b, c, d, e, f \in k$. Choose any irreducible representation $S$ over $\mathcal{C}$ such that $\chi(S)=\mathfrak{m}$ and $\operatorname{dim}_{k}(S)=n$. Let $C_{f}$ be the Clifford algebra associated to the binary cubic form $f(u, v)=a u^{3}+3 b u^{2} v+3 c u v^{2}+d v^{3}$. Therefore, there are algebra surjections

$$
\mathcal{C} \rightarrow \mathcal{C}_{f} \rightarrow \mathcal{C} / \mathfrak{m} \mathcal{C}
$$

where the first surjection sends $\Delta$ to a nonzero discriminant $D$ in $C_{f}$ (see Eq.(2)). By Theorem 2.7, $\mathcal{C}_{f}$ is an Azumaya algebra of PI degree three. So every irreducible representation over $\mathcal{C}_{f}$ is three-dimensional and the same holds for its image $\mathcal{C} / \mathfrak{m} \mathcal{C}$ as well. Since $\mathfrak{m} S=0$, we can view $S$ as an irreducible representation over $\mathcal{C} / \mathfrak{m} \mathcal{C}$. This implies that $\operatorname{dim}_{k}(S)=n=3$.

(b) follows from Theorem 2.2 and Lemma 4.2 since $\mathcal{C}$ has PI degree three.

(c) Let $S(a, b)=\mathcal{C} /(x-a, y-b)$ be any one-dimensional representation over $\mathcal{C}$ for some $a, b \in k$. One checks by Eq.(8) that

$$
\chi(S(a, b))=\left(z_{0}-a^{3}, z_{1}-a^{2} b, z_{2}-a b^{2}, z_{3}-b^{3}, z_{4}, z_{5}\right)=: \mathfrak{n} .
$$

Also we have $\chi(S(\omega a, \omega b))=\chi\left(S\left(\omega^{2} a, \omega^{2} b\right)\right)=\mathfrak{n}$. Suppose $\chi^{-1}(\mathfrak{n})=\left\{S_{1}, S_{2}, \ldots, S_{t}\right\}$. According to [8, Theorem 3.1(f)],

$$
\operatorname{dim}_{k}\left(S_{1}\right)+\operatorname{dim}_{k}\left(S_{2}\right)+\cdots+\operatorname{dim}_{k}\left(S_{t}\right) \leq \mathrm{PI}-\operatorname{deg}(\mathcal{C})=3
$$

Hence $t \leq 3$ and $\chi^{-1}(\mathfrak{n})$ exactly contains three one-dimensional irreducible representations $S(a, b), S(\omega a, \omega b)$ and $S\left(\omega^{2} a, \omega^{2} b\right)$. Moreover, $\chi$ maps $\operatorname{Irr}_{1} \mathcal{C}$ onto $\mathcal{S}_{\mathcal{C}}$ by Corollary 3.5, The result follows. 
(d) By (b), we have the surjection $\chi: \mathcal{C}=\operatorname{Irr}_{1} \mathcal{C} \sqcup \operatorname{Irr}_{3} \mathcal{C} \rightarrow Y=\mathcal{S}_{\mathcal{C}} \sqcup\left(Y \backslash \mathcal{S}_{\mathcal{C}}\right)$. By (c), we have an induced surjection $\chi: \operatorname{Irr}_{3} \mathcal{C} \rightarrow Y \backslash \mathcal{S}_{\mathcal{C}}$. Note that $\mathcal{C}$ has PI degree three. By the same argument as in $(\mathrm{c}), \chi$ yields a one-to-one correspondence between $\operatorname{Irr}_{3} \mathcal{C}$ and $Y \backslash \mathcal{S}_{\mathcal{C}}$.

(e) is a consequence of $(\mathrm{d})$ and $(\mathrm{b})$ of Theorem 2.2 .

4.5. As recalled in Theorem 2.7, Haile showed that the Clifford algebra $\mathcal{C}_{f}$ is an Azumaya algebra when the binary cubic form $f$ is nondegenerate or the discriminant $D \neq 0$ (see Eq.(2)). In the following, we give a necessary and sufficient condition for $\mathcal{C}_{f}$ to be Azumaya. It turns out that $\mathcal{C}_{f}$ can be an Azumaya algebra even when $f$ is degenerate or $D=0$.

4.6. Corollary. Let $k$ be a base field of characteristic different from 2 or 3 . The Clifford algebra $\mathcal{C}_{f}$ associated to the binary cubic form $f(u, v)=a u^{3}+3 b u^{2} v+3 c u v^{2}+$ $d v^{3}$ is an Azumaya algebra (of PI degree three) if and only if the point $(a, b, c, d)$ does not lie on the affine twisted cubic curve in $\mathbb{A}^{4}$ described in Lemma 3.2.

Proof. Without loss of generality, we can assume $k$ to be algebraically closed. Recall $Z=k\left[z_{0}, \ldots, z_{5}\right]$, where $z_{4}^{2}=z_{5}^{3}-27 \Delta$, is the center of $\mathcal{C}$. It is easy to check that $Z$ has a $k$-basis

$$
\left\{z_{0}^{i_{0}} z_{1}^{i_{1}} z_{2}^{i_{2}} z_{3}^{i_{3}} z_{4}^{i_{4}} z_{5}^{i_{5}} \mid 0 \leq i_{0}, i_{1}, i_{2}, i_{3}, i_{4}, 0 \leq i_{5} \leq 2\right\}
$$

As a consequence, it induces a projection $\pi: \operatorname{maxSpec}(Z) \rightarrow \mathbb{A}^{4}$ given by the natural inclusion $k\left[z_{0}, z_{1}, z_{2}, z_{3}\right] \hookrightarrow Z$. Therefore the natural surjection $\mathcal{C} \rightarrow \mathcal{C}_{f}$ factors through $\mathcal{C} / \mathfrak{m} \mathcal{C}$ for some $\mathfrak{m} \in \operatorname{maxSpec}(Z)$ if and only if $\mathfrak{m} \in \pi^{-1}(a, b, c, d)$. By Theorem 4.4, we know

$$
\mathcal{C} / \mathfrak{m} \mathcal{C} \cong \begin{cases}\mathrm{M}_{3}(k) & \mathfrak{m} \in \operatorname{maxSpec}(Z) \backslash S_{\mathcal{C}} \\ \text { a local algebra } & \mathfrak{m} \in S_{\mathcal{C}}\end{cases}
$$

By Corollary 3.5, $\pi^{-1}(a, b, c, d) \cap \mathcal{S}_{\mathcal{C}} \neq \emptyset$ if and only if $(a, b, c, d)$ lies in the affine twisted cubic curve in $\mathbb{A}^{4}$ given by Lemma 3.2. In this case, we know $\mathcal{C}_{f}$ has irreducible representations of both dimensions one and three. Otherwise, all the irreducible representations over $\mathcal{C}_{f}$ are of dimension three. Thus the result follows by the ArtinProcesi theorem on polynomial identities (see [1, Theorem 8.3]).

\section{Point VARiety of $\mathcal{C}$}

5.1. In noncommutative projective algebraic geometry (e.g. see [3]), a point module $M=\bigoplus_{i \geq 0} M_{i}$ over $\mathcal{C}$ is a cyclic graded module with Hilbert series $\frac{1}{1-t}$, or namely $M$ is generated by $M_{0}$ and $\operatorname{dim}_{k} M_{i}=1$ for all $i \geq 0$. Note that every point module $M$ over $\mathcal{C}$ can be denoted by the following diagram

$$
M\left(p_{0}, p_{1}, p_{2}, \ldots\right):
$$

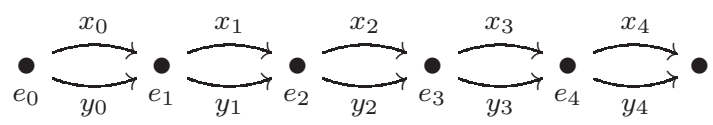


where $M=\bigoplus_{i \geq 0} k e_{i}$ and $0 \neq p_{i}=\left(x_{i}, y_{i}\right) \in k^{2}$ with the $\mathcal{C}$-action on $M$ given by

$$
x e_{i}=x_{i} e_{i+1}, \quad y e_{i}=y_{i} e_{i+1} .
$$

It is straightforward to check that two point modules $M\left(p_{0}, p_{1}, \ldots\right)$ and $M\left(q_{0}, q_{1}, \ldots\right)$ are isomorphic as graded $\mathcal{C}$ modules if and only if $p_{i}=q_{i}$ in $\mathbb{P}^{1}$ for all $i$ or $\left(p_{0}, p_{1}, \ldots\right)=$ $\left(q_{0}, q_{1}, \ldots\right)$ in $\mathbb{P}^{1} \times \mathbb{P}^{1} \times \cdots$. Therefore, the isomorphism classes of all point modules over $\mathcal{C}$ can be parametrized by a subvariety in $\mathbb{P}^{\infty}$, which is called the point variety of $\mathcal{C}$. Sometimes, the point variety in $\mathbb{P}^{\infty}$ is determined by a variety $X$ in $\mathbb{P}^{r}$ for some $r \geq 1$ and an automorphism $\sigma: X \rightarrow X$, where the point variety is determined by the natural embedding $X \hookrightarrow \mathbb{P}^{\infty}$ via $x \mapsto\left(x, \sigma(x), \sigma^{2}(x), \ldots\right)$ for any $x \in X$. In such a case for simplicity, we say the point variety is given by the pair $(X, \sigma)$.

5.2. Theorem. The point variety of $\mathcal{C}$ is given by the pair $\left(\mathbb{P}^{1} \times \mathbb{P}^{1} \times \mathbb{P}^{1}\right.$, id $)$.

Proof. Let $M\left(p_{0}, p_{1}, \ldots\right)$ be a point module. Taking any relation $f$ in Eq.(1) of $\mathcal{C}$, we have $f e_{0}=0$. Therefore we obtain

$$
\left\{\begin{aligned}
& x_{3} x_{2} x_{1} y_{0}-y_{3} x_{2} x_{1} x_{0}=0 \\
& x_{3} x_{2} y_{1} y_{0}+x_{3} y_{2} x_{1} y_{0}-y_{3} x_{2} y_{1} x_{0}-y_{3} y_{2} x_{1} x_{0}=0 \\
& x_{3} y_{2} y_{1} y_{0}-y_{3} y_{2} y_{1} x_{0}=0
\end{aligned}\right.
$$

Consider the above equations as a system of linear equations in terms of $x_{3}, y_{3}$ and rewrite it in the matrix form.

$$
\left[\begin{array}{cc}
x_{1} x_{2} y_{0} & -x_{0} x_{1} x_{2} \\
x_{2} y_{0} y_{1}+x_{1} y_{0} y_{2} & -x_{0} x_{2} y_{1}-x_{1} x_{0} y_{2} \\
y_{0} y_{1} y_{2} & -x_{0} y_{1} y_{2}
\end{array}\right]\left[\begin{array}{l}
x_{3} \\
y_{3}
\end{array}\right]=0
$$

It is easy to check that it has a solution $\left(x_{3}, y_{3}\right)=\left(x_{0}, y_{0}\right)$. We claim that it is the only solution up to a scalar multiple by showing that the coefficient matrix can not be identically zero. Suppose it is not true, then all the entries appearing in the $3 \times 2$ matrix must be zero. Hence

$$
x_{1} x_{2} y_{0}=x_{0} x_{1} x_{2}=x_{2} y_{0} y_{1}+x_{1} y_{0} y_{2}=x_{0} x_{2} y_{1}+x_{1} x_{0} y_{2}=y_{0} y_{1} y_{2}=x_{0} y_{1} y_{2}=0 .
$$

One can check the equations have no solution.

By repeating the above argument regarding the basis vector $e_{i}$ for all $i$, we see that $M\left(p_{0}, p_{1}, \ldots\right)$ is well defined if and only if $p_{i}=p_{i+3}$ for all $i$. This yields our result.

5.3. Let $\underline{p}=\left(p_{0}, p_{1}, p_{2}\right)$ be a point in $\mathbb{P}^{1} \times \mathbb{P}^{1} \times \mathbb{P}^{1}$. According to Theorem 5.2, the point variety over $\mathcal{C}$ is given by all $M(\underline{p})$ for some $\underline{p} \in \mathbb{P}^{1} \times \mathbb{P}^{1} \times \mathbb{P}^{1}$. Denote by $\Gamma=\left\{(p, p, p) \mid p \in \mathbb{P}^{1}\right\} \subset \mathbb{P}^{1} \times \mathbb{P}^{1} \times \mathbb{P}^{1}$ the diagonal part.

5.4. Theorem. Let $M(\underline{p})$ be any point module over $\mathcal{C}$, and $S$ be a simple quotient of $M(p)$.

(a) If $\underline{p} \in \Gamma$, then $\operatorname{dim}_{k}(S)=1$. 
(b) If $\underline{p} \notin \Gamma$, then $S$ is either trivial or $\operatorname{dim}_{k}(S)=3$.

Proof. Let $M=M\left(p_{0}, p_{1}, p_{2}\right)=\bigoplus_{i \geq 0} k e_{i}$. We have $\mathcal{C}$ acts on $M$ as in (11).

(a) Suppose $p_{0}=p_{1}=p_{2}=(a, b) \in \mathbb{P}^{1}$. It is direct to check that the central elements $\left\{z_{i}\right\}_{0 \leq i \leq 5}$ in Eq.(8) act on $M$ as follows.

$$
z_{0} e_{i}=a^{3} e_{i+3}, z_{1} e_{i}=a^{2} b e_{i+3}, z_{2} e_{i}=a b^{2} e_{i+3}, z_{3} e_{i}=b^{3} e_{i+3}, z_{4} e_{i}=z_{5} e_{i}=0 .
$$

Hence $\left(z_{0} z_{3}-z_{1} z_{2}, z_{0} z_{2}-z_{1}^{2}, z_{1} z_{3}-z_{2}^{2}, z_{4}, z_{5}\right) \subset \operatorname{Ann}_{\mathcal{C}}(M) \subset \operatorname{Ann}_{\mathcal{C}}(S)$. By Theorem 4.4 and Corollary 3.5, we have $\mathbb{V}\left(\operatorname{Ann}_{\mathcal{C}}(S) \cap Z\right) \in \mathcal{S}_{\mathcal{C}}$. Hence $\operatorname{dim}_{k} S=1$.

(b) According to Eq.(8), we obtain the following actions on $M$.

$$
\left(z_{0} z_{3}-z_{1} z_{2}\right) e_{i}=-\frac{a}{9} e_{i+6},\left(z_{0} z_{2}-z_{1}^{2}\right) e_{i}=-\frac{b}{18} e_{i+6},\left(z_{1} z_{3}-z_{2}^{2}\right) e_{i}=-\frac{c}{18} e_{i+6},
$$

where the coefficients $a, b, c$ are given by

$$
\begin{aligned}
& a=x_{2} y_{2}\left(x_{1} y_{0}-x_{0} y_{1}\right)^{2}+x_{1} y_{1}\left(x_{2} y_{0}-x_{0} y_{2}\right)^{2}+x_{0} y_{0}\left(x_{2} y_{1}-x_{1} y_{2}\right)^{2} \\
& b=x_{0}^{2}\left(x_{2} y_{1}-x_{1} y_{2}\right)^{2}+x_{1}^{2}\left(x_{0} y_{2}-x_{2} y_{0}\right)^{2}+x_{2}^{2}\left(x_{1} y_{0}-x_{0} y_{1}\right)^{2} \\
& c=y_{0}^{2}\left(x_{2} y_{1}-x_{1} y_{2}\right)^{2}+y_{1}^{2}\left(x_{0} y_{2}-x_{2} y_{0}\right)^{2}+y_{2}^{2}\left(x_{1} y_{0}-x_{0} y_{1}\right)^{2} .
\end{aligned}
$$

Here we write $p_{i}=\left(x_{i}, y_{i}\right) \in \mathbb{P}^{1}$ for all $i \geq 0$. Now suppose $\operatorname{dim}_{k} S=1$ and it is not trivial. By Theorem 4.4 and Corollary 3.5, we have $\left(z_{0} z_{3}-z_{1} z_{2}, z_{0} z_{2}-z_{1}^{2}, z_{1} z_{3}-z_{2}^{2}\right) \subset$ $\operatorname{Ann}_{\mathcal{C}}(S)$. If any of $a, b, c$ is not zero, it implies that $e_{i+6}=0$ in $S$ for all $i$. Thus $x e_{5}=y e_{5}=0$ in $S$. Hence $e_{5}=0$ in $S$ otherwise $S=k e_{5}$ would be trivial. Repeating the argument, we obtain all $e_{i}=0$ in $S$. It is absurd. So $a=b=c=0$. Now set the variables

$$
X:=\left(x_{1} y_{0}-x_{0} y_{1}\right)^{2}, Y:=\left(x_{2} y_{0}-x_{0} y_{2}\right)^{2}, Z:=\left(x_{2} y_{1}-x_{1} y_{2}\right)^{2} .
$$

We write the above conditions as a system of linear equations in terms of $X, Y, Z$ as follows.

$$
\left[\begin{array}{ccc}
x_{2} y_{2} & x_{1} y_{1} & x_{0} y_{0} \\
x_{2}^{2} & x_{1}^{2} & x_{0}^{2} \\
y_{2}^{2} & y_{1}^{2} & y_{0}^{2}
\end{array}\right]\left[\begin{array}{c}
X \\
Y \\
Z
\end{array}\right]=0 .
$$

We will show that Eq.(12) has no solution whenever $p \notin \Gamma$, which yields that $\operatorname{dim}_{k} S>$ 1 if $S$ is not trivial. Hence part (b) follows from Theorem 4.4. Note that $X=0$ implies that $p_{0}=p_{1}, Y=0$ implies that $p_{0}=p_{2}$, and $Z=0$ implies that $p_{1}=p_{2}$. Since $p \notin \Gamma,(X, Y, Z)$ is a nonzero solution for Eq.(12). So

$$
0=\operatorname{det}\left(\begin{array}{ccc}
x_{2} y_{2} & x_{1} y_{1} & x_{0} y_{0} \\
x_{2}^{2} & x_{1}^{2} & x_{0}^{2} \\
y_{2}^{2} & y_{1}^{2} & y_{0}^{2}
\end{array}\right):=\gamma
$$

Direct computation shows that $\gamma^{2}=X Y Z$. So $\mathbb{V}(\gamma)=\mathbb{V}(X) \cup \mathbb{V}(Y) \cup \mathbb{V}(Z)$. Without loss of generality, we assume that $p \in \mathbb{V}(\gamma) \cap \mathbb{V}(X)$. Since $X=0$, we know $\left(x_{0}, y_{0}\right)=\left(x_{1}, y_{1}\right) \in \mathbb{P}^{1}$. Moreover, we can check that $Y=Z \neq 0$. But in such a 
case, $(X, Y, Z)$ does not satisfy Eq.(12). We obtain a contradiction, which proves our result.

\section{Discriminant ideals of $\mathcal{C}$}

6.1. Discriminant ideals of PI algebras play an important role in the study of a maximal orders [16], the automorphism and isomorphism problems for noncommutative algebras [9], the Zariski cancellation problem for noncommutative algebras [4], and the description of dimensions of irreducible representations 8 .

6.2. Let $A$ be an algebra and $C \subseteq Z(A)$ be a central subalgebra. A trace map on $A$ is a nonzero map $\operatorname{tr}: A \rightarrow C$ that is cyclic $(\operatorname{tr}(x y)=\operatorname{tr}(y x)$ for $x, y \in A)$ and $C$-linear. For a positive integer $\ell$, the $\ell$-th discriminant ideal $D_{\ell}(A / C)$ and the $\ell$-th modified discriminant ideal $M D_{\ell}(A / C)$ of $A$ over $C$ are the ideals of $C$ with generating sets

$$
\left\{\operatorname{det}\left(\left[\operatorname{tr}\left(y_{i} y_{j}\right)\right]_{i, j=1}^{\ell}\right) \mid y_{1}, \ldots, y_{\ell} \in A\right\}
$$

and

$$
\left\{\operatorname{det}\left(\left[\operatorname{tr}\left(y_{i} y_{j}^{\prime}\right)\right]_{i, j=1}^{\ell}\right) \mid y_{1}, y_{1}^{\prime}, \ldots, y_{\ell}, y_{\ell}^{\prime} \in A\right\} .
$$

6.3. Since we know the binary cubic generic Clifford algebra $\mathcal{C}$ is Auslander-regular, Cohen-Macaulay, and stably-free (it is connected graded), we can employ Stafford's work [17, Theorem 2.10] to conclude that $\mathcal{C}$ is a maximal order in a central simple algebra and admits the reduced trace map tr $: \mathcal{C} \rightarrow Z$ (e.g. [16, Section 9]). The next theorem describes the zero sets of the discriminant ideals of $\mathcal{C}$ which has PI degree three.

6.4. Theorem. Let $\mathcal{C}$ be the binary cubic generic Clifford algebra of PI degree three with reduced trace map $\operatorname{tr}: \mathcal{C} \rightarrow Z$. For all positive integers $\ell$, the zero sets of the $\ell$-th discriminant and $\ell$-th modified discriminant ideals of $\mathcal{C}$ over its center $Z$ coincide, $\mathbb{V}\left(D_{\ell}(\mathcal{C} / Z), \operatorname{tr}\right)=\mathbb{V}\left(M D_{\ell}(\mathcal{C} / Z)\right.$, tr $)$; denote this set by $\mathbb{V}_{\ell} \subseteq Y:=\max \operatorname{Spec}(Z)$. The following hold:

(1) $\mathbb{V}_{\ell}=\emptyset$ for $\ell \leq 3$

(2) $\mathbb{V}_{\ell}=Y^{\text {sing }}=\mathcal{S}_{\mathcal{C}}$ for $4 \leq \ell \leq 9$.

(3) $\mathbb{V}_{\ell}=Y$ for $\ell>9$.

Proof. For any $\mathfrak{m} \in \operatorname{maxSpec}(Z)$, denote by $\operatorname{Irr}_{\mathfrak{m}}(\mathcal{C})$ the set of isomorphism classes of irreducible representations of $\mathcal{C}$ with central annihilator $\mathfrak{m}$. By [8, Main Theorem (a),(e)], we have

$\mathbb{V}\left(D_{\ell}(\mathcal{C} / Z), \operatorname{tr}\right)=\mathbb{V}\left(M D_{\ell}(\mathcal{C} / Z), \operatorname{tr}\right)=\left\{\mathfrak{m} \in \operatorname{maxSpec}(Z) \mid \sum_{V \in \operatorname{Irr}_{\mathfrak{m}}(\mathcal{C})}\left(\operatorname{dim}_{k} V\right)^{2}<\ell\right\}$.

Hence, our result follows directly from Theorem 4.4, 


\section{REFERENCES}

[1] M. Artin, On Azumaya algebras and finite dimensional representations of rings, J. Algebra II (1969), 532-563.

[2] M. Artin and W. Schelter, Graded algebras of global dimension 3, Adv. Math. 66 (1987), $171-216$.

[3] M. Artin, J. Tate, and M. Van den Bergh, Some algebras associated to automorphisms of elliptic curves, The Grothendieck Festschrift Vol. I, 33-85, Birkhäuser Boston, Boston, MA, 1990.

[4] J. Bell and J. J. Zhang, Zariski cancellation problem for noncommutative algebras, Selecta Math. (N.S.), 23 (2017), 1709-1737.

[5] G. Bergman, The diamond lemma for ring theory, Adv. Math. 109 (2) (1994), 228-287.

[6] K. A. Brown and K. R. Goodearl, Lectures on algebraic quantum groups, Advanced Courses in Mathematics. CRM Barcelona, Birkhäuser, Basel (2002).

[7] K. A. Brown and K. R. Goodearl, Homological aspects of noetherian PI Hopf algebras and irreducible modules of maximal dimension, J. Algebra, 198 (1997), 240-265.

[8] K. A. Brown and M. T. Yakimov, Azumaya loci and discriminant ideals of PI algebras, Preprint, 2017. Available at http://arxiv.org/abs/1702.04305v4.

[9] S. Ceken, J. H. Palmieri, Y.-H. Wang, and J. J. Zhang, The discriminant controls automorphism groups of noncommutative algebras, Adv. Math., 269 (2015), 551-584.

[10] K. Chan, A. A. Young, and J. J. Zhang, Discriminant formulas and applications, Algebra Number Theory, 10 (2016), 557-596.

[11] K. R. Goodearl and R. B. Warfield, Jr., An Introduction to Noncommutative Noetherian Rings, Second Edition, London Mathematical Society Student Texts 61, Cambridge University Press, Cambridge, 2004.

[12] D. E. Haile, On the Clifford algebra of a binary cubic form, Amer. J. Math., 106 (6) (1984), 1269-1280.

[13] R. Hartshorne, Algebraic Geometry, Berlin, Heidelberg, New York: Springer-Verlag, 1977.

[14] N. Heerema, An algebra determined by a binary cubic form. Duke Math. J., 21 (1954), 423-443.

[15] J. C. McConnell and J. C. Robson, Noncommutative Noetherian Rings Graduate Studies in Mathematics 30, American Mathematical Society, Providence, 2000.

[16] I. Reiner, Maximal orders, volume 28 of London Mathematical Society Monographs. New Series. The Clarendon Press, Oxford University Press, Oxford, 2003. Corrected reprint of the 1975 original, With a foreword by M. J. Taylor.

[17] J. T. Stafford, Auslander-regular algebras and maximal orders, J. London Math. Soc. (2), 50 (1994), 276-292.

[18] C. Ure and R. S. Kulkarni, The Generic Clifford Algebra, privately circulated, Algebra Extravaganza, July 2017, Temple University, Philadelphia.

[19] S.-Q. Wang and Q.-S. Wu, A class of AS-regular algebras of dimension five, J. of Algebra, 362 (2012), 117-144.

E-mail address: 1hwang@pitt.edu

Department of Mathematics, University of Pittsburgh, Pittsburgh, PA 15260

E-mail address: xingting.wang@howard.edu

Department of Mathematics, Howard University, Washington, D.C. 20059 